\title{
Excessive left ventricular hypertrophy in moderate degenerative aortic stenosis: an ineffective compensatory mechanism triggered by primary myocardial dysfunction and enhanced by concomitant mild renal impairment?
}

\author{
Dorota Długosz ${ }^{1}$, Klaudiusz Bolt ${ }^{1}$, Wai S. Sam ${ }^{1}$, Tomasz Nawara ${ }^{1}$, Olga Kruszelnicka ${ }^{2 *}$, \\ Bernadeta Chyrchel ${ }^{3 *}$, Andrzej Surdacki ${ }^{3 *}$ \\ 'Students' Scientific Group at the $2^{\text {nd }}$ Department of Cardiology, Jagiellonian University Medical College, Krakow, Poland \\ ${ }^{2}$ Department of Coronary Artery Disease and Heart Failure, John Paul II Memorial Specialist Hospital, Krakow, Poland \\ ${ }^{3} 2^{\text {nd }}$ Department of Cardiology, Jagiellonian University Medical College, Krakow, Poland \\ *Joint senior authors on this work (SSG tutors)
}

\section{INTRODUCTION}

Recent progress has been focused on the management of severe degenerative aortic stenosis (AS), while its antecedent, moderate AS, still poses a clinical challenge due to the considerable impact of non-valvular factors on its pathophysiology and presentation [1]. Importantly, the prognosis in moderate AS is worse than commonly assumed [2].

Left ventricular (LV) hypertrophy (LVH) develops gradually in moderate AS and has traditionally been perceived as an adaptive mechanism to preserve LV systolic function. However, in hypertension [3, 4] and AS [5, 6], inappropriately high LV mass (iLVM), i.e. an excess of LV mass (eLVM) greater than $28 \%$ to $35 \%$ of the theoretical LV mass (LVM) predicted from the individual haemodynamic load in a reference population $[3,4]$, was associated with adverse outcome and depressed afterload-corrected LV midwall fractional shortening (mwFS). The latter is a better index of LV performance than ejection fraction (EF) that overestimates LV function in concentric LVH [7].

Although the prevalence of iLVM was increasingly high and was associated with deteriorating renal function in chronic kidney disease (CKD) and hypertension [8, 9], patients with renal disease had been excluded from key reports on determinants and prognostic value of iLVM in AS [5, 6]. Thus, our aim was to assess relations between renal function, eLVM, and mwFS in real-world patients with moderate AS.

\section{METHODS}

We retrospectively analysed medical records of 150 consecutive patients diagnosed with moderate AS (aortic valve area [AVA] 1.0-1.5 $\mathrm{cm}^{2}$ ) [10] at discharge, who where hospitalised in a cardiology department. Seventy patients in a stable clinical condition entered the final analysis. The exclusion criteria included more than mild aortic regurgitation or disease of another valve, atrial fibrillation, $\mathrm{EF}<40 \%$, unstable in-hospital serum creatinine level, glomerular filtration rate (GFR) $<15 \mathrm{~mL} / \mathrm{min} / 1.73 \mathrm{~m}^{2}$ estimated by the CKD-EPI formula, and relevant non-cardiac comorbidities (except for well-controlled diabetes).

The study protocol was approved by the Ethics Committee of the Jagiellonian University (approval no. 122.6120.228.2016).

Based on data extracted from in-hospital echocardiographic records, we calculated the LVM (by modified Devereux formula), relative LV wall thickness (RWT), and mwFS (assuming a constant LV wall volume throughout the cardiac cycle [7]). From echocardiography and averaged in-hospital blood pressure (BP), circumferential end-systolic

\section{Address for correspondence:}

Andrzej Surdacki, MD, PhD, $2^{\text {nd }}$ Department of Cardiology, Faculty of Medicine, Jagiellonian University Medical College, ul. Kopernika 17,

31-501 Kraków, Poland, tel/fax: +48 1242471 80, e-mail: surdacki.andreas@gmx.net

Received: 7.08.2018 Accepted: 29.08.2018 Available as AoP: 17.09.2018

Kardiologia Polska Copyright (c) Polish Cardiac Society 2018 
Table 1. Echocardiographic characteristics, left ventricular (LV) afterload, and excess of LV mass according to glomerular filtration rate (GFR)

\begin{tabular}{|c|c|c|c|c|c|}
\hline \multirow[t]{2}{*}{ Characteristic } & \multicolumn{3}{|c|}{ GFR $\left[\mathrm{mL} / \mathrm{min} / 1.73 \mathrm{~m}^{2}\right]$} & \multirow[t]{2}{*}{$\mathbf{p}$} & \multirow[t]{2}{*}{$p$ for trend } \\
\hline & $\begin{array}{c}>85 \\
(n=18)\end{array}$ & $\begin{array}{c}60-85 \\
(n=30)\end{array}$ & $\begin{array}{c}15-59 \\
(n=22)\end{array}$ & & \\
\hline Aortic valve area $\left[\mathrm{cm}^{2}\right]$ & $1.2 \pm 0.1$ & $1.2 \pm 0.1$ & $1.2 \pm 0.2$ & 0.5 & 0.8 \\
\hline LV diastolic diameter $[\mathrm{cm}]$ & $5.1 \pm 0.7$ & $4.9 \pm 0.6$ & $4.9 \pm 0.7$ & 0.7 & 0.5 \\
\hline LV mass $[\mathrm{g}]$ & $178 \pm 49$ & $203 \pm 45$ & $207 \pm 60$ & 0.2 & 0.15 \\
\hline LV mass/volume ratio $[\mathrm{g} / \mathrm{mL}]$ & $1.49 \pm 0.38$ & $1.85 \pm 0.46^{*}$ & $1.85 \pm 0.50^{*}$ & 0.02 & 0.04 \\
\hline Relative LV wall thickness & $0.42 \pm 0.09$ & $0.49 \pm 0.10^{*}$ & $0.49 \pm 0.10^{*}$ & 0.04 & 0.07 \\
\hline Ejection fraction [\%] & $61 \pm 8$ & $59 \pm 7$ & $60 \pm 6$ & 0.7 & 0.9 \\
\hline LV midwall fractional shortening [\%] & $17 \pm 3$ & $14 \pm 4^{*}$ & $15 \pm 4^{*}$ & 0.03 & 0.12 \\
\hline cESS $[\mathrm{hPa}]$ & $181 \pm 65$ & $147 \pm 69$ & $163 \pm 65$ & 0.4 & 0.4 \\
\hline Valvulo-arterial impedance $\left[\mathrm{mmHg} / \mathrm{mL} / \mathrm{m}^{2}\right]$ & $4.2 \pm 1.4$ & $5.4 \pm 2.6$ & $5.0 \pm 1.5$ & 0.15 & 0.1 \\
\hline Excess of LV mass [\%] & $-2(-6-13)$ & $21(1-57)^{*}$ & $61(-18-85)^{*}$ & 0.03 & 0.01 \\
\hline
\end{tabular}

Data are shown as mean \pm standard deviation or median (interquartile range). cESS — circumferential end-systolic LV wall stress ${ }^{*} p<0.05$, vs. GFR $>85 \mathrm{~mL} / \mathrm{min} / 1.73 \mathrm{~m}^{2}$ by post-hoc Fisher test or Mann-Whitney test.

LV wall stress (cESS) [7], valvulo-arterial impedance, a joint estimate of valvular and arterial factors that oppose ventricular ejection $\left(\mathrm{Zva}\left[\mathrm{mmHg} / \mathrm{mL} / \mathrm{m}^{2}\right]=\right.$ (systolic BP + mean aortic gradient) / stroke volume index) [11], and eLVM were computed. The latter is a percentage deviation from the predicted LVM, where LVM ${ }_{\text {predicted }}[\mathrm{g}]=55.37+\{6.63 \times$ (height $\left.[\mathrm{m}])^{2.7}\right\}+\{0.009216 \times($ stroke volume $[\mathrm{mL}]) \times($ systolic $\mathrm{BP}+$ peak aortic gradient $[\mathrm{mmHg}])\}-(18.1 \times \mathrm{n})$, with $\mathrm{n}=1$ for men and $\mathrm{n}=2$ for women [3-6].

\section{Statistical analysis}

Patients' characteristics were compared according to the categories of GFR (derived from averaged measurements of serum creatinine during the index hospitalisation by the CKD-EPI formula: group A [GFR > 85], B [GFR: 60-85], and C [GFR: 15-59]) by analysis of variance (ANOVA), Kruskal-Wallis ANOVA, or $\chi^{2}$ test, and trend effects were determined by Spearman's coefficients. Additionally, semi-standardised regression coefficients $(\beta)$ were computed for univariate and multivariate predictors of eLVM and mwFS, using multiple linear ridge regression due to collinearity between candidate predictors, including age, sex, coronary artery disease, GFR, AVA, Zva, cESS, and RWT. A p-value $<0.05$ was assumed as significant.

\section{RESULTS AND DISCUSSION}

Excess of LVM and age increased gradually across decreasing GFR categories (Table 1; Suppl. Table S1; Suppl. Fig. S1 - see journal website). Compared to group A, subjects from groups B and C had significantly higher systolic BP, RWT, and LVM/volume ratio (Suppl. Fig. S2 - see journal website), and lower LV mwFS (Suppl. Fig. S3 - see journal website), all of which were similar in patients from groups $\mathrm{B}$ and $\mathrm{C}$ (Table 1).

After Bonferroni correction for multiple comparisons, eLVM was positively associated with age, Zva, RWT, and $\mathrm{LVM}$ /volume ratio and inversely with GFR, mwFS, and cESS. Also, mwFS correlated negatively with RWT and LVM/volume ratio (all unadjusted $p$-values $\leq 0.001$ ) (Suppl. Table S2 - see journal website).

In multivariate analysis, the inverse eLVM-mwFS relationship was strengthened, and eLVM was associated only with mwFS $(\beta=-36 \pm 6, \mathrm{p}<0.001)$ and $\mathrm{cESS}$ $(\beta=-29 \pm 6, \mathrm{p}<0.001)$, whereas mwFS was related to $\operatorname{eLVM}(\beta=-2.6 \pm 0.4, \mathrm{p}<0.001)$ and $\operatorname{cESS}(\beta=-1.9 \pm 0.4$, $p<0.001)$. Substitution of LVM/volume ratio for RWT did not change the results.

We found that progressive early GFR decline coincided with increasing eLVM and reduced mwFS in moderate AS. Importantly, upon multivariate adjustment, these associations lost their significance, while the inverse eLVM-mwFS relationship was further strengthened. Additionally, body mass index, previously linked to iLVH $[4,6]$, was similar across groups A-C. As AVA, cESS, and LV end-diastolic diameter (a raw estimate of LV preload) were similar irrespective of GFR, we hypothesised that gradual development of excessive LVH could be triggered by load-independent primary myocardial dysfunction and amplified by coexistent renal impairment. This mechanism might keep cESS relatively constant despite disease progression, but does not ensure the restoration of LV performance, which may explain progressive eLVM increase across groups $\mathrm{A}-\mathrm{C}$ and similar reduction in mwFS in groups $B$ and $C$ vs. $A$. 
The concept of iLVM in AS a compensatory (albeit ineffective) mechanism evoked by depressed LV contractility, not elevated LV afterload, was first proposed by Italian researchers, who found lower mwFS for each given level of cESS in patients with iLVM [5, 6], in analogy to hypertension [3, 4]. However, patients with renal disease had been excluded from those AS studies [5, 6]. Thus, our results supplement an earlier report of increasingly high prevalence of iLVM and associated depressed mwFS along CKD stages 2-5, beginning at mild renal dysfunction [9] in mainly hypertensive subjects without coexisting AS. Therefore, our study is the first to show early renal impairment as a contributor to eLVM in AS.

Because eLVM independently predicted adverse outcome in severe AS [5], it seems to be a potential therapeutic target. However, renin-angiotensin system inhibitors (RASI), a mainstay for nephroprotection and LVH inhibition, are frequently avoided in AS due to fear of hypotension. Nevertheless, the use of RASI was associated with the lack of adverse prognostic effects and slower LVM progression in mild-to-moderate AS [12].

In conclusion, early renal impairment enhances excessive LVH that appears secondary to subtle, load-independent LV systolic dysfunction. Prevention of early kidney deterioration in less-than-severe AS may slow development of excessive $\mathrm{LVH}$ and improve long-term prognosis.

\section{Acknowledgements}

The results of this study were presented at the $22^{\text {nd }}$ International Congress of the Polish Cardiac Society (Krakow, Poland) and the $22^{\text {nd }}$ Annual Scientific Meeting of the Heart Failure Society of America (Nashville, USA). The study was supported by Jagiellonian University Medical College (No. K/ZDS/006105 — to A.S.).

\section{Conflict of interest: none declared}

\section{References}

1. Stewart WJ. Aortic stenosis is still very tricky, especially when it is moderate. J Am Coll Cardiol. 2017; 69(19): 2393-2396, doi: 10.1016/j.jacc.2017.03.569, indexed in Pubmed: 28494977.
2. Yechoor P, Blaustein AS, Bakaeen FG, et al. The natural history of moderate aortic stenosis in a veteran population. J Thorac Cardiovasc Surg. 2013; 145(6): 1550-1553, doi: 10.1016/j. jtcvs.2012.05.013, indexed in Pubmed: 22664178.

3. de Simone G, Palmieri V, Koren MJ, et al. Prognostic implications of the compensatory nature of left ventricular mass in arterial hypertension. J Hypertens. 2001; 19(1): 119-125, indexed in Pubmed: 11204291.

4. Palmieri V, Wachtell K, Gerdts E, et al. Left ventricular function and hemodynamic features of inappropriate left ventricular hypertrophy in patients with systemic hypertension: the LIFE study. Am Heart J. 2001; 141(5): 784-791, doi: 10.1067/mhj.2001.114803, indexed in Pubmed: 11320367.

5. Cioffi G, Faggiano P, Vizzardi E, et al. Prognostic effect of inappropriately high left ventricular mass in asymptomatic severe aortic stenosis. Heart. 2011; 97(4): 301-307, doi: 10.1136/hrt.2010.192997, indexed in Pubmed: 20720251.

6. Cioffi G, de Simone G, Cramariuc D, et al. Inappropriately high left-ventricular mass in asymptomatic mild-moderate aortic stenosis. J Hypertens. 2012; 30(2): 421-428, doi: 10.1097/HJH.0b013e32834fob00, indexed in Pubmed: 22179090.

7. de Simone G, Devereux RB, Roman MJ, et al. Assessment of left ventricular function by the midwall fractional shortening/end-systolic stress relation in human hypertension. J Am Coll Cardiol. 1994; 23(6): 1444-1451, indexed in Pubmed: 8176105.

8. Nardi E, Palermo A, Mulè G, et al. Left ventricular hypertrophy and geometry in hypertensive patients with chronic kidney disease. J Hypertens. 2009; 27(3): 633-641, doi: 10.1097/HJH.0b013e3283220ecd, indexed in Pubmed: 19262231.

9. Cioffi G, Tarantini L, Frizzi R, et al. Chronic kidney disease elicits excessive increase in left ventricular mass growth in patients at increased risk for cardiovascular events. J Hypertens. 2011; 29(3): 565-573, doi: 10.1097/HJH.0b013e3283424188, indexed in Pubmed: 21150636.

10. Baumgartner H, Falk V, Bax J, et al. [2017 ESC/EACTS Guidelines for the management of valvular heart disease]. Kardiol Pol. 2018; 76(1): 1-62, doi: 10.5603/KP.2018.0013.

11. Briand M, Dumesnil JG, Kadem L, et al. Reduced systemic arterial compliance impacts significantly on left ventricular afterload and function in aortic stenosis: implications for diagnosis and treatment. J Am Coll Cardiol. 2005; 46(2): 291-298, doi: 10.1016/j. jacc.2004.10.081, indexed in Pubmed: 16022957.

12. Bang CN, Greve AM, Rossebø AB, et al. Renin-angiotensin system inhibition is not associated with increased sudden cardiac death, cardiovascular mortality or all-cause mortality in patients with aortic stenosis. Int J Cardiol. 2014; 175(3): 492-498, doi: 10.1016/j.ijcard.2014.06.013, indexed in Pubmed: 25012498.

Cite this article as: Długosz D, Bolt K, Sam WS, et al. Excessive left ventricular hypertrophy in moderate degenerative aortic stenosis: an ineffective compensatory mechanism triggered by primary myocardial dysfunction and enhanced by concomitant mild renal impairment? Kardiol Pol. 2018; 76(10): 1486-1488, doi: 10.5603/KP.a2018.0196. 